\title{
Exclusión social y muerte súbita cardiaca
}

\author{
Social exclusion and sudden cardiac death
}

\section{Luis Alberto Ochoa Montes}

Especialista de II Grado en Medicina Interna y Cardiología. Máster en Ciencias en Investigación en Aterosclerosis. Hospital Clinicoquirúrgico "Hermanos Ameijeiras". La Habana, Cuba.

\section{RESUMEN}

Anualmente fallecen en los llamados países del primer mundo de forma inesperada debido a enfermedades del corazón y los vasos sanguíneos, entre 350000 y 400 000 personas según reportes oficiales. Esto equivale a 1000 muertes súbitas cada día. La situación de salud en 31 de los 35 países del continente americano, donde las cardiopatías constituyen la primera causa de muerte, no dista mucho de lo observado en Occidente y Estados Unidos y es en general un problema que sigue creciendo. Existen factores de tipo médico que deben ser tomados en cuenta al analizar el comportamiento del fenómeno en cada uno de los países pero existen también factores de índole política de abordaje impostergable para lograr resultados favorables en la disminución de la morbilidad y mortalidad por enfermedades cardiovasculares y finalmente, en la mortalidad súbita. Se profundiza en la vinculación existente entre exclusión social y enfermedad cardiovascular y se plantea la interrogante de si la exclusión social juega un papel determinante en la elevada incidencia de muerte súbita cardiaca en la era actual. La respuesta a esta interrogante solo puede ser encontrada en el sistema económico, político y social que impere en las naciones. Solo globalizando las oportunidades de atención médica, incluyendo a los que tienen poco o nada tienen, no excluyendo, otorgando a todos acceso a las nuevas tecnologías en el campo de la medicina, se puede afrontar el reto del incremento de la pandemia cardiovascular en el siglo XXI y por ende, de la muerte súbita.

Palabras clave: Muerte súbita cardiaca, exclusión social, enfermedad cardiovascular, aterosclerosis. 


\begin{abstract}
In the so-called first world countries, 350000 to 400000 people die suddenly from heart and blood vessel diseases, according to official data. This means 1000 sudden deaths every day. The health situation in 31 out of the 35 countries in the American continent, where heart diseases are the first cause of death, is not very far from the situation in the United States and the Western nations since this is generally a growing problem. There are some medical factors that should be taken into account when analyzing the behaviour of this phenomenon in each country, but there are also political factors that must be approached to accomplish favourable results in the reduction of morbidity and mortality from cardiovascular diseases and of sudden cardiac mortality. The linking of social exclusion and cardiovascular disease is deeply studied. The answer to the question of whether the social exclusion presently plays a determining role in the high incidence of sudden cardiac death or not can only be found in the economic, political and social system existing in the nations. The globalization of the medical care opportunities by including rather than excluding those who have a little or nothing at all - giving everybody access to the new technologies in the medical field - is the means to face the challenge of the rise of cardiovascular pandemic in the 21st century, and hence, of the sudden death.
\end{abstract}

Key words: Sudden cardiac death, social exclusion, cardiovascular disease, atherosclerosis.

\title{
INTRODUCCIÓN
}

\section{Definición de muerte súbita cardiaca}

Es una muerte natural debido a causas cardiacas, anunciada por pérdida de conciencia brusca, que se produce en el plazo de $1 \mathrm{~h}$, tras el comienzo de los síntomas agudos, en un individuo que se sabe presenta una cardiopatía preexistente, conocida o no por el paciente, pero el tiempo y modo de la muerte son inesperados. ${ }^{1,2}$

\section{Magnitud del problema}

Anualmente fallecen en los llamados países del primer mundo de forma inesperada debido a enfermedades del corazón y los vasos sanguíneos, según reportes oficiales entre 350000 y 400000 personas. Esto equivale a 1000 muertes súbitas cada día y un evento por cada minuto en que transcurre la lectura de este artículo. La situación de salud en 31 de los 35 países del continente americano, donde las cardiopatías constituyen la primera causa de muerte, no dista mucho de lo observado en Occidente y Estados Unidos. ${ }^{3,4}$ El flagelo de la muerte súbita cobra cada día más victimas que la pandemia de VIH/sida, tuberculosis o cáncer. La situación epidemiológica reciente por el virus de la influenza A (H1N1) que obligó a la Organización Mundial de la Salud (OMS) a decretar el 11 de junio del 2009 la Fase 6 de alerta (pandemia) y ya se habían notificado oficialmente 96911 casos en 133 países, con 436 fallecidos, ${ }^{5}$ no ha ocasionado en toda su evolución los índices 
de letalidad que el fallecimiento inesperado en solo $24 \mathrm{~h}$. Su alcance es solo comparable con el efecto de un golpe terrorista a gran escala "Morir súbitamente del corazón es como sufrir un ataque terrorista similar al 11 de Septiembre de 2001 contra el World Trade Center de Nueva York, cada 72 horas."

El impacto de este fenómeno sigue in crescendo. Su magnitud rebasa el marco del número de eventos que se manifiestan anualmente, de los cuales las dos terceras partes ocurren fuera del ámbito hospitalario., ${ }^{4,6-8}$ Las victimas llegan a los sistemas de urgencias y emergencias hospitalarios con registros electrocardiográficos que denotan el fin de la vida. ${ }^{9,10}$ El dramatismo que le acompaña: la pérdida de un ser humano, en no pocas ocasiones de forma prematura, en edades jóvenes, en sujetos aparentemente sanos debido a un trastorno del ritmo cardiaco, ${ }^{11,12}$ donde "la muerte acontece como un rayo en un cielo despejado", le dan una connotación amarga dado lo inestimable de estas perdidas en el marco familiar, económico y social.

El desconsuelo que traduce una muerte súbita y las innumerables interrogantes que alrededor del suceso se generan, solo contribuyen a aumentar las dudas y la sensación de pesimismo en los familiares y amigos de la victima.

Múltiples y diversos son los factores que condicionan esta realidad de hoy. Factores de tipo médicos, como son los relacionados con la prevalencia de la enfermedad arterial coronaria en los diferentes países, responsable del $90 \%$ de las muertes súbitas de causas cardiacas, la existencia o ausencia de registros estadísticos oficiales para analizar el alcance de este flagelo, la ausencia de una definición aceptada por los diferentes especialistas (internistas, cardiólogos, epidemiólogos, patólogos, legistas) sobre todo al abordar el tiempo que debe transcurrir para que el suceso sea considerado como súbito, sin duda alguna el punto más álgido en la definición, y el considerar como deceso súbito a los casos no recuperados con diagnóstico de fibrilación ventricular, cuando en la práctica de cada 3 casos diagnosticados se obtiene un registro eléctrico.

Estos factores deben ser tomados en cuentas al analizar el comportamiento del fenómeno en cada uno de los países. ${ }^{4,7,13-15}$

Pudiera surgir la pregunta:

¿Son estos factores "médicos", los determinantes de esta triste realidad de hoy en los países del llamado primer mundo y las naciones pobres al encarar el progresivo ascenso de esta pandemia de muerte súbita cardiovascular?

Factores de índole políticos determinantes, son de abordaje impostergable en virtud de lograr resultados favorables en la disminución de la morbilidad y mortalidad por enfermedades cardiovasculares y como objetivo final la mortalidad súbita.

\section{EXCLUSIÓN SOCIAL Y ENFERMEDAD CARDIOVASCULAR}

La exclusión social, la cual se traduce en ausencia de oportunidades para todos; en el acápite que se analiza: la salud, la pobreza, la marginalidad, el analfabetismo y la implantación de modelos consumistas que promueven el tener por encima del ser, el yo por delante del nosotros, contribuyen a que el desarrollo de la medicina y los resultados de la revolución científico-técnica no sean por igual extendidos a las diferentes capas sociales. 
Los costos por concepto de atención médica de urgencia ante una dolencia cardiaca, y la toma de decisiones en relación a "conductas intervencionistas", llevan aparejado la condición social de quien la sufre y no la condición humana, pilar esencial para cualquier modelo que ponga por delante al ser humano.

"...un ataque cardíaco es una ventana para evaluar los efectos de la clase social en la atención diferenciada de salud", aseveró J. Scott en su Editorial del 23 de Mayo de 2005 en el New York Times, a propósito de la historia de tres neoyorquinos de diferentes estratos sociales, que solo tuvieron en común presentar una enfermedad coronaria aguda. A pesar de lo irracional que resulta, en cada caso "la solución médica al episodio coronario" no estuvo en relación con el número de vasos coronarios comprometidos, la situación hemodinámica del paciente, ni las enfermedades concomitantes al momento de ocurrir el evento, todo lo contrario, fue la condición social de las victimas la que determinó el tiempo que medió en la atención de urgencia, nivel de cobertura hospitalaria ofrecida, costos, tipo de terapia y rehabilitación recibida. Posterior al cuadro coronario agudo, el pronóstico y la calidad de vida de los tres pacientes, nunca fue la misma.

El $90 \%$ de los eventos de muerte súbita cardiaca se manifiestan en la población general con factores de riesgo que propician el desarrollo de algún tipo de cardiopatía. La obesidad, diabetes mellitus, inactividad física, hipertensión arterial y las dislipidemias, ${ }^{3,16,17}$ por solo citar algunos, constituyen verdaderas pandemias en el siglo XXI, alimentadas por el consumismo (comida chatarra, abuso del desarrollo tecnológico en detrimento del régimen de actividad física, patrones culturales de sociedades desarrolladas, pérdida de estándares y condiciones de vida y el estrés crónico imperativo de la vida moderna) y lo más grave: "La exportación de estos vicios a los llamados países del tercer mundo o subdesarrollados" o "transculturación", con las lógicas consecuencias que trae aparejado.

Citando el editorial del The New York Times, Santos Gracia en su editorial titulado: Salud y Clases Sociales en América. ¿Sueño o pesadilla?, publicado unos meses después, realiza un análisis del problema, en lo que considera una relación inversa entre el tipo de educación y los ingresos per cápita, con la letalidad por insuficiencia cardiaca, ictus, diabetes y algunos tipos de tumores.

\section{Santos Gracia señala que:}

Factores como el tabaquismo, dieta de mala calidad, poca actividad física, obesidad, hipertensión, hipercolesterolemia y stress, son más frecuentes entre los menos favorecidos por la educación y los ingresos, también son los peores candidatos para permitirse un cambio de estilo de vida que los alejen de un posible ataque cardiaco. ${ }^{18}$

Estos factores son la base de lo que vendrá después: el desarrollo de la aterosclerosis, principal depredador del ser humano al ser el mayor responsable de la aparición de alguna forma de daño cardiovascular. ${ }^{19-22}$ La modificación de hábitos y patrones de vida no cardiosaludables expresan la esencia de la prevención primaria. ${ }^{3,19}$

Hasta este punto vale la pregunta:

¿Cómo podrán contribuir los sistemas económicos que promueven la exclusión social y el consumismo a evitar el desarrollo de la enfermedad cardiovascular?

Una vez que está presente el daño al corazón y los vasos sanguíneos, es necesario trazar estrategias de estratificación de riesgo para la prevención de futuros 
eventos, instaurar los tratamientos más eficaces para evitar la progresión de la enfermedad, disminuir las recurrencias y lograr la reinserción a la sociedad al individuo enfermo, con calidad de vida (prevención secundaria). Estas medidas logran disminuir la mortalidad general y también la de forma súbita. ${ }^{8,23,24}$

Principios como la accesibilidad, gratuidad, una relación costo-beneficio adecuada, y lo que es más importante, ofrecer las mejores terapias con menos riesgos para el paciente, son acciones necesarias para evitar la muerte súbita.

Otra interrogante sería:

¿La accesibilidad a este tipo de atención estará garantizada por igual para todos los pacientes, con independencia del estrato social y la condición económica de que sea partícipe?

La muerte súbita cardiaca representa la forma de presentación del $25 \%$ de los síndromes coronarios agudos. Uno de cada dos pacientes con afecciones cardiovasculares muere súbitamente, las dos terceras partes en el medio extrahospitalario y de ellos un tercio en ausencia de testigos presénciales. ${ }^{24,25}$

A partir de estos elementos, al presentarse la parada cardiaca (3ra. etapa de la muerte súbita), la prontitud con que se realice el rescate de la victima, por un sistema de emergencia médica y la instauración de medidas de apoyo vital que garanticen el mantenimiento de la vida, unido a la terapia más efectiva médica o quirúrgica, disminuirán significativamente la mortalidad. ${ }^{22,23}$

En la cardiopatía isquémica aguda, responsable del $90 \%$ de los decesos súbitos (15 $\%$ en las primeras $6 \mathrm{~h}$ por fibrilación ventricular), acceder a un tratamiento revascularizador, que logre restituir el flujo sanguíneo coronario mediante trombolisis coronaria, angioplastia coronaria transluminal percutánea [ACTP] con colocación de Stein vs. cirugía de revascularización, disminuye sensiblemente la mortalidad general y particularmente, la súbita. ${ }^{26}$ Los trastornos del ritmo cardiaco ocasionan la mayor mortalidad prematura, atribuible a arritmias ventriculares malignas (fibrilación ventricular primaria o secundaria), por lo cual se requiere del internamiento de las victimas, una vez rescatadas a la mayor brevedad, en unidades de cuidados intensivos coronarios. ${ }^{27-29}$

Continuaba argumentando Santos Gracia, en su editorial, la relación entre el tipo de educación y los ingresos personales, al aseverar que los peores candidatos para ser resucitados e ingresar en las unidades de cuidados intensivos son aquellos individuos no favorecidos socialmente, o sea, excluidos por el modelo impuesto a los países latinoamericanos. ${ }^{18}$

El haber presentado un primer episodio de muerte súbita incrementa potencialmente el riesgo de un segundo evento (si el episodio sucede en el medio extrahospitalario, el riesgo aumenta), ${ }^{8,22}$ por lo que es vital la estratificación de riesgo del paciente en el transcurso de las primeras $24 \mathrm{~h}$, para establecer el pronóstico y conducta ulterior.

Evaluar la presencia de isquemia residual, inestabilidad eléctrica y disfunción ventricular izquierda (marcadores de muerte súbita cardiaca en la isquemia aguda) son pilares necesarios e indicativos de la terapéutica definitiva en las victimas de deceso inesperado. Esto conlleva al traslado de los supervivientes a laboratorios de hemodinamia y electrofisiología para estos fines. . $^{3,30,31}$

Cabe preguntarse:

http://scielo.sld.cu 
¿Puede La exclusión social a las que conduce irremediablemente el modelo consumista capitalista, determinar el tipo de atención, pronóstico y tratamiento de los pacientes que han sido rescatados con vida de una muerte súbita cardíaca?

Luego de la estratificación del riesgo (riesgo alto-indeterminado) en quien ha sufrido una parada cardiaca, es necesario optar en el menor tiempo posible por una terapia definitiva. Los grupos de alto e indeterminado riesgo se benefician de un dispositivo implantable que prevenga nuevas crisis de arritmias ventriculares malignas, el desfibrilador automático implantable (DAI). Solo un grupo minoritario muy particular se beneficia con terapias específicas como son los marcapasos, ablación física o quirúrgica de vías anómalas, dispositivos de resincronización ventricular y otros. ${ }^{3,17}$

Una vez más, es necesario preguntarse:

¿Las diferencias en accesibilidad, inmediatez, calidad en los servicios de atención a la salud, costos, recursos disponibles, a que conduce la crisis neoliberal-consumista de un modelo que protege mayoritariamente a las minorías con posibilidades de recibir este tipo de atención, podrá contribuir a lograr la mayor utopía que en la primera década del actual siglo aspiran los que se dedican a atender a enfermos cardiovasculares con este tipo de evento: disminuir la incidencia de la muerte súbita cardiaca ?

Lo expuesto hasta aquí, permite formular otra pregunta:

¿La exclusión social de la que son victimas irremediablemente millones de seres humanos en el planeta jugará un papel determinante en la elevada incidencia de muerte súbita cardiaca en la era actual?

Está claro que las respuestas a estas interrogantes con independencia de la ideología que se tenga, no pueden ser encontradas en otra fuente que no sea el sistema económico-político y por ende social que impere en las naciones. Las políticas de los gobiernos a favor de la preservación de la salud y la vida en el planeta, "voluntad política", deben estar dirigidas a brindar una atención médica de excelencia con el menor riesgo para el paciente, sin reparar en el poder económico, condición social, religión, credo, raza, sexo o ideología de quien sufre la pérdida de la salud.

No es posible ganar esta batalla si solo se limita a ver "la arista médica"del problema (punta del iceberg), cuando se trata del enfrentamiento a un problema de complejidad mayor. En las sociedades de consumo su esencia queda expresada en el binomio: ser $y$ tener.

Hay que ganar conciencia de que los gobiernos son los verdaderos responsables de la suerte de sus pueblos, por lo tanto se deben a ellos. La "voluntad política"de los gobiernos por revertir esta realidad compartida deberá ser un común denominador para todas las naciones. Solo de esta forma se podrá afrontar el enorme reto a que conduce la pandemia cardiovascular en el siglo XXI.

Lo indicado es encaminar los esfuerzos a globalizar los recursos para el desarrollo, a globalizar la fe, a globalizar la esperanza y solo con la concepción de que este problema global se debe enfrentar globalizando las oportunidades de atención médica, incluyendo a los que tienen poco o nada tienen, no excluyendo, otorgando acceso a las nuevas tecnologías en el campo de la medicina y no bloqueando el acceso en nombre de la condición social a que destina el modelo neoliberal. 
La pobreza, la ignorancia fruto del analfabetismo y la exclusión social de un sistema egoísta por naturaleza no tendrá que ser necesariamente el mejor futuro que soñamos para la humanidad.

Estamos convencidos que un mundo mejor es posible.

Para comprender lo enunciado anteriormente es necesario partir de un análisis elemental a que conduce la siguiente interrogante.

¿Qué debe ser lo primario para un modelo económico-social: El ser o el tener?

Cualquiera que sea la respuesta como convicción del modelo y los valores que defendamos, debe expresar más...

Debe expresar el compromiso con las nuevas generaciones.

Debe llevar el legado impostergable a los que vendrán después.

Debe tener como reto final: la supervivencia de la especie humana.

Esto determinará en última instancia el resto y es lo que permite plantear la relación que existe entre: Exclusión Social y Muerte Súbita Cardiaca.

\section{REFERENCIAS BIUBLIOGRÁFICAS}

1. Fitzgerald K. ¿Qué es la muerte súbita? [sitio en Internet]. [citado 2009].

Disponible en: http://www.aeped.es/gepmsl/protocolos/libro blanco/anexo16.pdf

2. Wilson JD, Braunwald E, Isselbacher KJ, Petersdoff RD. Colapso cardiovascular, Parada cardiaca y Muerte Súbita. En: Harrison TR, editor. Principios de Medicina Interna. 15ta ed. Madrid: Interamericana Mc Graw- Hill; 2005. p. 282-8.

3. Myerburg RJ. Sudden cardiac death: exploring the limits of our knowledge. J Cardiovasc Electrophysiol. 2002;12:369-81.

4. Corrons J. Revisión sobre muerte súbita cardíaca. Rev Esp Cardiol. 1997;40:1313.

5. Actualización-Epidemiología Influenza A (H1N1). Situación Epidemiológica -6 de julio del 2009 [sitio en Internet]. [citado 2009]. Disponible en:

http://www.sld.cu/sitios/influenzaporcina/

6. Sanz G. Muerte súbita. Paro cardiorrespiratorio. En: Farreras $P$, editor. Medicina Interna. 15ta ed. Barcelona: Mosby/Doyma Libros; 2004. p. 407.

7. Robert JM, Castellanos A. Cardiac arrest and sudden cardiac death. In: Braunwald, editor. Heart disease. $5^{\text {th }}$ ed. Filadelfia: Saunders; 1997. p. 742-72.

8. Ochoa Montes LA, González Lugo M, Tamayo Vicente ND, Romero del Sol JM, Correa Azahares DP, Miguélez Nodarse $R$, et al. El ámbito de la Parada Cardiaca como determinante en el Pronóstico de aparición de la Muerte Súbita Cardíaca. 
Revista electrónica Portales Médicos [sitio en Internet]. Nov 2008 [citado 2009];III(20). Disponible en: http://www.portalesmedicos.com

9. . Arritmias Finales en la Muerte Súbita Cardíaca. Revista electrónica Portales Médicos [sitio en Internet]. Oct 2008 [citado 2009];III(19). Disponible en: http://www.portalesmedicos.com

10. Heikki V, Huikiri Castellanos A. Sudden death due to cardiac arrhytmias. NEJM. $2001 ; 345: 1473-85$.

11. Bayés de Luna A, Guindo Soldevilla J. Muerte súbita de origen cardíaco. Rev Esp Cardiol. 1998;51:330-7.

12. Marrugat J. Elosua R, Gil M. Epidemiología de la muerte súbita cardíaca en España. Rev Esp Cardiol. 1999;52:717-25.

13. Friedman M, Manwaring JH, Cobb LA. Resuscitation from out-of-Hospital ventricular fibrillation: four-year follow- up. Circulation. 1975;51-52(supl 3):223.

14. Siscovick DS. Challenges in cardiac arrest research. Ann Emerg Med. $1993 ; 22: 92$.

15. Rodríguez Font E, Viñolas Prat X. Muerte Súbita Cardíaca (III). Causas de Muerte Súbita. Problemas a la hora de establecer y clasificar los tipos de Muerte. Rev Esp Cardiol. 1999; 52:1004-14.

16. Myerburg RJ. Sudden cardiac death: Structure, function, and time dependence of risk. Circulation. 1992;85(supl 1):1-2.

17. Myerburg RJ, Castellanos A. Parada cardiaca y muerte súbita cardiaca. En: Braunwald E, editor. Tratado de Cardiología. Madrid: Interamericana Mc Graw- Hill; 2006. p. 1087-139.

18. Santos Gracia J. Salud y Clases Sociales en América. ¿Sueño o pesadilla? LATIDOS. 2006;Año 2(2).

19. Ochoa Montes LA, González Lugo M, Tamayo Vicente ND, Romero del Sol JM, Rodríguez Hernández N, Fernández-Britto Rodríguez JE. Aterosclerosis, Riesgo vascular y Muerte Súbita Cardíaca. Revista electrónica Portales Médicos [sitio en Internet]. Nov 2008 [citado 2009];III(6). Disponible en:

http://www.portalesmedicos.com

20. Fernández-Britto JE, Castillo Herrera JA. Aterosclerosis. Rev Cubana Invest Biomed [sitio en Internet]. 2005[citado 2008];24(3). Disponible en:

http://scielo.sld.cu/scielo.php?script=sci_arttext\&pid=S0864-

$03002005000300001 \& \operatorname{lng}=$ es [editorial]

21. Fuster V, Ros R, Topol E. Aterosclerosis y enfermedad arterial coronaria. Berlín: Springerverlag Iberia; 1997.

22. López Messa JB. ¿Debe el paro cardiaco extrahospitalario ser una Enfermedad de Declaración Obligatoria? Artículo especial A No.86. Revista Electrónica de Medicina Intensiva [sitio en Internet]. 2008 [citado 2009];8(5). Disponible en: http://remi.uninet.edu 
23. Nichol G, Rumsfeld J, Eigel B. Essential Features of Designating Out-of-Hospital cardiac Arrest as a Reportable Event. Circulation. 2008;117:2299-308.

24. Zipes DP, Wellen HJJ. Sudden Cardiac death. Circulation. 1998(21):2334-51.

25. Ochoa Montes La Muerte Súbita del Corazón. Granma. 18 Jun 2007;Secc. Consulta Médica:2.

26. Adgey AA, Devlin JE, Webb SW, Mulholland HC. Initiation of ventricular fibrillation outside hospital in patients with acute ischemic heart disease. $\mathrm{BHJ}$. 1982;47:55.

27. Cox JL, Daniel TM, Boineau JP. The Electrophysiologic time course of acute myocardial ischemia and the effects of early coronary artery reperfusion. Circulation. 1973;48:971.

28. Cooper RS, Simmons BE, Castaner A. Left ventricular hypertrophy is associated with worse survival independent of ventricular function and number of coronary arteries severely narrowed. Am J Cardiol. 1990;65:441.

29. Kimura S, Bassett AL, Kohya T. Simultaneous recording of action potential from endocardium and epicardium during ischemia in the isolated cat ventricle: relation of temporal electrophysiological heterogeneities to arrhythmias. Circulation. $1986 ; 74: 401$.

30. Coronel R, Wilms-Schopman FJG, Opthof T. Reperfusion arrhythmias in isolated perfused pig hearts: in homogeneities in extra-cellular potassium. ST and QT potentials and transmembrana action potential. Circ Res. 1992;71:1131.

31. Bayés-Genis A, Viñolas X, Guindo J, Fiol M, Bayés de Luna A.

Electrocardiography and clinical precursors of ventricular fibrillation: chain of events. J Cardiovasc Electrophysiol. 1995; 6:410-7.

Recibido: 30 de julio de 2009.

Aprobado: 2 de febrero de 2010.

Luis Alberto Ochoa Montes. Hospital Clinicoquirúrgico "Hermanos Ameijeiras". Sección B, Manzana 9, No.7. Reparto "Frank País". Arroyo Naranjo. La Habana, Cuba. E-mail: ochoam@infomed.sld.cu 